Montana Tech Library

Digital Commons@ Montana Tech

Center for Advanced Mineral and Metallurgical

Processing (CAMP)

Faculty Scholarship

4-15-1971

\title{
Ion-Pair Theory of Concentrated Electrolytes. IV. Ion Atmosphere Charge Distribution
}

Frank Stillinger

Bell Telephone Laboratories

Ronald White

Montana Tech of the University of Montana

Follow this and additional works at: https://digitalcommons.mtech.edu/camp

Part of the Chemistry Commons, and the Physics Commons

\section{Recommended Citation}

F.H. Stillinger and R.J. White. Ion-pair theory of concentrated electrolytes: IV. ion atmosphere charge distribution. J. Chem. Phys. 54:3405-3411, 1971.

This Article is brought to you for free and open access by the Faculty Scholarship at Digital Commons @ Montana Tech. It has been accepted for inclusion in Center for Advanced Mineral and Metallurgical Processing (CAMP) by an authorized administrator of Digital Commons @ Montana Tech. For more information, please contact sjuskiewicz@mtech.edu. 


\title{
Ion-Pair Theory of Concentrated Electrolytes. IV. Ion Atmosphere Charge Distribution
}

\author{
Frank H. Stillinger and Ronald J. White* \\ Bell Telephone Laboratories, Incorporated, Murray Hill, New Jersey 07974
}

(Received 21 December 1970)

\begin{abstract}
Arguments are presented indicating that the large-size limiting behavior of the ion-pair size distribution is qualitatively the same both with, and without, Coulomb interactions between ions. This in turn implies that the static dielectric response function $\epsilon_{0} / \epsilon(k)$ derived in the preceding paper is nonanalytic at $k=0$. The specific singular behavior of this response requires a branch cut along the imaginary $k$ axis. It furthermore induces an $r^{-8}$ tail in the ion atomsphere charge distribution at finite electrolyte concentration.
\end{abstract}

\section{INTRODUCTION}

The preceding paper ${ }^{1}$ provides an expression for the static dielectric response function $\epsilon(k)$ in the primitive model symmetrical electrolyte, i.e., uniformly charged rigid-sphere ions suspended in a dielectric continuum solvent. In this model there are only two independent ionic pair correlation functions,

$$
g_{++}{ }^{(2)}(r) \equiv g_{--}{ }^{(2)}(r), \quad g_{+-}{ }^{(2)}(r) \equiv g_{-+}{ }^{(2)}(r),
$$

and their difference is proportional to the radial density of electrostatic charge in the electrolyte's ion atmospheres. One of the basic relationships in the foregoing theory is an integral transform linking this difference to $\epsilon(k)$ :

$$
\begin{aligned}
g_{+-}(2)(r)-g_{++}{ }^{(2)}(r)=\left(2 \pi^{2} c r\right)^{-1} \\
\times \int_{0}^{\infty} k \sin (k r)\left\{\frac{k^{2}}{\kappa^{2}}\left[\frac{\epsilon_{0}}{\epsilon(k)}-1\right]+1\right\} d k ;
\end{aligned}
$$

in this expression $c$ is the anion (or cation) number density, $\epsilon_{0}$ stands for the solvent dielectric constant (independent of $k$ ), and $\kappa$ is the usual Debye-Hückel parameter:

$$
\kappa^{2}=8 \pi c(Z e)^{2} / k_{B} T \epsilon_{0} .
$$

Investigation of the ion atmosphere structure using Eq. (1.2) will be our primary objective in this article.

The preceding $\epsilon(k)$ calculation $^{1}$ was based upon a complete pairing of all ions to form "dipolar molecules," whose orientation preference in external fields could then self-consistently be calculated. The size distribution function $p^{(1)}(s)$ for these ion pairs plays an important role in the formalism, and in fact $\epsilon(k)$ is a functional of $p^{(1)}(s)$. Through Eq. (1.2), the large- $r$ nature of ion atmospheres depends on the small- $k$ behavior of $\epsilon(k)$, and this in turn requires knowledge of the large-s regime in $p^{(1)}(s)$. The following section argues that the "tail" of $p^{(1)}$ is substantially the same for the electrolyte as for uncharged spheres.

Section III subsequently demonstrates that $\epsilon(k)$ is not analytic at $k=0$ in the complex $k$ plane. This nonanalyticity is connected with a branch cut of $\epsilon(k)$, and leads through Eq. (1.2) to a relatively long-ranged contribution to the ion atmosphere charge density proportional to $r^{-8}$. This behavior is at variance with the orthodox predictions that exponential damping should occur, in particular in the domain of applicability of the Debye-Hückel theory. ${ }^{2}$ One can show, however, that this algebraic contribution does not affect the DebyeHückel limit.

On account of the unconventional character of our algebraic ion atmosphere prediction, Sec. IV briefly reviews alternative electrolyte theories, to establish what weaknesses within them might cause one to overlook such a prediction. In particular we mention the Ursell-Mayer cluster techniques, and the integral equations that are often used to predict correlation functions. In each case it seems that approximations employed in these alternative theories assure from the outset that exponential damping obtains, whether or not the true physical situation demands it.

Naturally we must ultimately be prepared to admit that a spurious approximation in the foregoing $\epsilon(k)$ computation $^{1}$ might be the source of our algebraic ion atmosphere falloff. Nevertheless, it seems valuable to have raised the general issue of spatial decay rates, and to have accented the inability of present electrolyte theory to decide this matter rigorously. Until suitable exact arguments become available, Monte Carlo calculations on the primitive model $^{3}$ perhaps could contribute to this aspect of the theory.

\section{CHARGE DISTRIBUTION NEAR AN ION PAIR}

The process of identifying ion pairs in the symmetrical electrolyte is implemented sequentially. Given a specific configuration $\mathbf{r}_{1} \cdots \mathbf{r}_{2 N}$ of the $N$ anions and $N$ cations in volume $V$, one forms a pair always by selecting the smallest anion-cation pair distance among those ions not already paired. This rule eventually exhausts all ions, each of which then has a unique partner of opposite charge sign. ${ }^{4}$

There will of course be $N$ ! different ways that ions could be paired with partners, if ions are considered to be distinguishable. But since all ions of given charge are mechanically identical, it suffices in analysis of equilibrium electrolyte properties merely to restrict attention to that portion of configuration space $(1 / N$ ! of the total) corresponding to a fixed pairing. In order to be consistent with the sequential pairing process, this restriction prevents oppositely charged ends of two distinct ion pairs from approaching each other more 


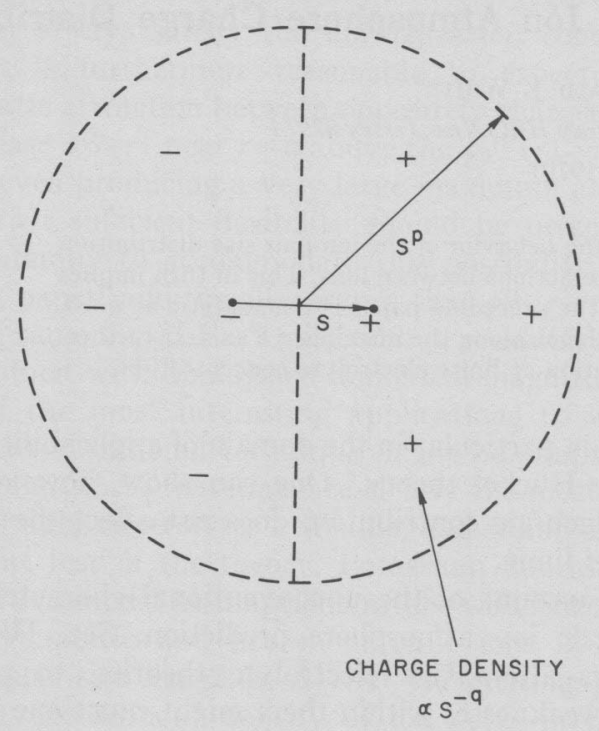

FIG. 1. Rough charge distribution near a fixed ion pair used in the scaling estimates in Sec. II. In each of the two hemispheres, the charge density is regarded as constant.

closely than the lesser of the two ion pair anion-tocation distances. ${ }^{5,6}$ Ion pairs may therefore be treated as stable "diatomic molecules" (with variable bond length) which interact not only via the original charged rigid-sphere forces, but through the infinitely repulsive "steric hindrance" forces generated by the single pairing restriction.

We denote the probability for anion-cation center distance $s$ in the ion pairs by $p^{(1)}(s)$, with the following normalization convention:

$$
c=4 \pi \int_{0}^{\infty} s^{2} p^{(1)}(s) d s
$$

The dipole moment $\mathbf{m}$ of an ion pair is Zes at any instant, so the mean square moment will be given by the integral

$$
\left\langle m^{2}\right\rangle=4 \pi(Z e)^{2} c^{-1} \int_{0}^{\infty} s^{4} p^{(1)}(s) d s .
$$

This integral converges when all ions are discharged $(Z e=0)$, since then $p^{(1)}(s)$ behaves for large $s$ thus, ${ }^{6}$

$$
\lim _{Z e \rightarrow 0} p^{(1)}(s)=p_{0}^{(1)}(s) \sim \text { const } \times s^{-6} .
$$

The effect of electrostatic interactions is to draw opposite ends of each ion pair together, which surely will decrease the spatial extension of $p^{(1)}(s)$. The mean square moment expression (2.2) therefore continues to remain finite for the electrolyte with fully charged ions.

The steric hindrance repulsions allow anions to accumulate around the anionic end of a given ion pair, and cations to accumulate around its cationic end. As a result, the surroundings of the pair will possess an average charge density whose own dipole moment $\mathrm{m}^{\dagger}(\mathrm{s})$ will be parallel to $\mathrm{m}$ by symmetry, and will change with size $s$.

The fact that the electrolyte is an electrical conductor implies:

$$
\lim _{k \rightarrow 0} \epsilon(k)=\infty ;
$$

in other words, long-wavelength components of an electrostatic field are completely shielded. The Kirkwood theory of polar dielectrics ${ }^{7}$ provides an expression for $\epsilon(k=0)$ which, in concert with phenomenological result (2.4), implies ${ }^{5}$

$$
\begin{aligned}
\langle\mathrm{m} \cdot \mathrm{m}\rangle & =4 \pi Z e c^{-1} \int_{0}^{\infty} s^{3} m^{\dagger}(s) p^{(1)}(s) d s \\
& =+\infty
\end{aligned}
$$

At first glance one might think that $p^{(1)}(s)$ would become exponentially damped by Coulomb interactions, analogous to the ion atmosphere damping that is prescribed by the Debye-Hückel theory. ${ }^{2}$ However, if $p^{(1)}(s)$ were to incorporate, say, the venerable factor $\exp (-\kappa s)$, then Eq. (2.5) would require the moment $m^{\dagger}(s)$ to diverge at least as fast as $\exp (+\kappa s)$ as $s \rightarrow \infty$. Such a rapidly increasing moment for large $s$ would either require an enormous local charge density (which would involve an impossibly high energy), or else would require ionic correlation over impossibly large distances. We must therefore claim that $p^{(1)}(s)$ retains an algebraic tail as the Coulomb interactions are switched on.

Theoretically it should be possible to use the variational principle in the preceding paper ${ }^{1}$ to support this claim, but so far we have been unable to produce the required analysis. Instead, it should suffice for present purposes to appeal to rough scaling arguments. Figure 1 illustrates the basis for that kind of analysis. We assume that the charge surrounding a pair is confined to the interior of a sphere centered on the ion pair. This sphere expands as $s$ increases, with radius proportional to $s^{p}$, $p \geq 1$. Within each hemisphere, the average charge is taken to be constant, with a value proportional to $s^{-q}$, $q \geq 0$. Since concern lies mainly with the large-s regime, the radius- $a$ exclusion spheres surrounding both ends of the fixed ion pairs can be completely disregarded.

It is easy to see how $m^{\dagger}(s)$ must vary since it involves essentially just the product of total charge in one hemisphere $\left(\propto s^{3 p-q}\right)$ times the distance between hemisphere centroids $\left(\propto s^{p}\right)$ :

$$
m^{\dagger}(s) \propto s^{4 p-q} .
$$

In order that this form produce the required divergence in Eq. (2.5), we must presumably have

$$
4 p-q \geq 2 \text {. }
$$

We must also assure that the electrostatic energy of the postulated charge distribution remain within reasonable bounds. This energy may be obtained from the standard expression

$$
E=\frac{1}{2} \int \rho \psi d \mathbf{r}
$$


in terms of charge density $\rho$ and potential $\psi$. The potential will scale according to the product of hemisphere charge and the inverse of the linear dimension $s^{p}$, at least where $\rho$ is nonvanishing. Thus,

$$
E \propto s^{5 p-2 q} .
$$

If this energy were grossly to exceed the thermal energy $k_{B} T$, it seems plausible that a spontaneous ionic rearrangement should occur to lower that energy. Thus the exponent in (2.9) should not in fact exceed zero:

$$
5 p-2 q \leq 0, \quad p \leq \frac{2}{5} q .
$$

The region in the $p, q$ plane that is consistent with the initially admissible values of these parameters, as well as inequalities (2.7) and (2.10), is shown in Fig. 2. This region is an infinite wedge whose vertex corresponds to simultaneous equality in (2.7) and (2.10) $\left(p=\frac{4}{3}, q=\frac{10}{3}\right)$.

The next step is to estimate the axial electrostatic force $\mathrm{F}$ exerted on the ion pair by the surrounding diffuse charge. Since $p>1$, the pair will be centrally located in a much bigger sphere as $s \rightarrow \infty$, so we need only to estimate the electric field strength at this center. This field will of course be proportional to hemisphere charge divided by the square of the characteristic linear dimension $s^{p}$ :

$$
|\mathbf{F}| \propto s^{p-q} .
$$

Inequalities (2.7) and (2.10) imply that

$$
p-q \leq-2 \text {. }
$$

Though the electrical force tends to drive the two ions of the pair together, its effect obviously diminishes no more slowly as $s$ increases than the direct attraction between those two ions.

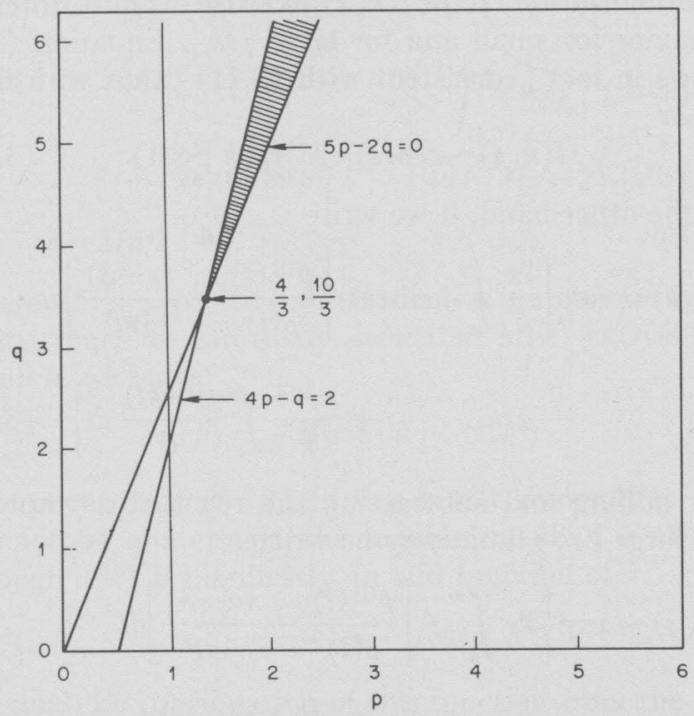

FIG. 2. Admissible region (shaded) for pairs of exponents $p, q$ describing local charge density around a fixed ion pair.
The direct Coulomb force in the ion pair, plus the indirect force (2.11) may be integrated with respect to $s$ to yield an electrostatic potential of mean force. The corresponding Boltzmann factor:

$$
\exp \left\{C\left(s_{0}\right)-\left(k_{B} T\right)^{-1} \int_{s_{0}}^{s}\left[\frac{(Z e)^{2}}{\epsilon_{0} t^{2}}+F(t)\right] d t\right\}
$$

would then multiply the uncharged sphere size distribution function $p_{0}^{(1)}(s)$ to convert it to the electrolyte function $p^{(1)}(s) .{ }^{8}$ According to Eqs. (2.11) and (2.12), this Boltzmann factor approaches a constant as $s \rightarrow \infty$. Therefore, $p^{(1)}(s)$ will be proportional to $s^{-6}$ in this limit. ${ }^{1,6}$

In contrast with the indirect force result (2.11)(2.12), an exponentially damped $p^{(1)}(s)$ would have required a force that approached a nonzero constant as $s \rightarrow \infty$.

Since the scaling argument which leads to the $s^{-6}$ behavior for $p^{(1)}(s)$ is rather crude, it is desirable to confirm its conclusion independently. The preceding paper $^{1}$ provides the following representation for $p^{(1)}(s)$ :

$$
p^{(1)}(s)=c^{2} \Gamma(s) /\left[1+4 \pi c \int_{a}^{s} t^{2} \Gamma(t) d t\right]^{2} .
$$

$\Gamma(s)$ is a correlation function that arises during the pairing process. At stage $s$ in the process, where all pairs of size smaller than $s$ have been formed, the concentration of unpaired anions or cations throughout the entire system will be

$$
\rho(s)=4 \pi \int_{s}^{\infty} t^{2} p^{(1)}(t) d t .
$$

However, the local density of anions at distance $s$ from an unpaired cation will be $\Gamma(s) \rho(s)$ by definition.

It is clear that for uncharged spheres $\Gamma(s)$ approaches a nonzero constant as $s \rightarrow \infty$, to assure the existence of the $s^{-6}$ tail for $p_{0}{ }^{(1)}(s)$. If $p^{(1)}(s)$ for charged ions were exponentially damped, it follows that $\Gamma(s)$ would have to increase exponentially fast with $s$. This would entail an extreme tendency for anions to "pile up" immediately outside the $s$ sphere surrounding the unpaired cation. Such an extreme accretion of oppositely charged ions would require a very powerful electrostatic driving force which, however, is not available.

\section{DIELECTRIC FUNCTION BRANCH POINT}

The inverse relation for Eq. (1.2) :

$$
\begin{aligned}
\frac{\epsilon_{0}}{\epsilon(k)}= & 1-\frac{\kappa^{2}}{k^{2}}\left\{1-\frac{4 \pi c}{k}\right. \\
& \left.\times \int_{0}^{\infty} r \sin (k r)\left[g_{+}-{ }^{(2)}(r)-g_{++}{ }^{(2)}(r)\right] d r\right\}
\end{aligned}
$$

may be used to show that the Debye-Hückel point ion linear theory implies

$$
\epsilon_{0} / \epsilon(k)=k^{2} /\left(k^{2}+\kappa^{2}\right),
$$


a meromorphic function in the complex $k$ plane. For large $|k|$, this result leads to

$$
\epsilon_{0} / \epsilon(k)=1-\left(\kappa^{2} / k^{2}\right)+\left(\kappa^{4} / k^{4}\right)-\cdots .
$$

By contrast, we may integrate Eq. (3.1) by parts with explicit consideration of the hard-sphere diameter $a$ to obtain the exact large- $|k|$ development for the primitive model:

$$
\begin{aligned}
{\left[\epsilon_{0} / \epsilon(k)\right] \sim 1-} & \left(\kappa^{2} / k^{2}\right)+\left(4 \pi c \kappa^{2} a / k^{4}\right) \cos (k a) \\
& \times\left[g_{+}{ }^{(2)}(a)-g_{++}{ }^{(2)}(a)\right]-\cdots .
\end{aligned}
$$

The trigonometric factor $\cos (k a)$ indicates that the point at infinity is really an essential singularity, rather than a regular point as the Debye-Hückel point-ion result (3.3) implies.

It is natural to inquire whether the exact dielectric response function differs in other fundamental ways from the analytic structure suggested by the DebyeHückel expression (3.2). We shall specifically examine the analytic character in the neighborhood of $k=0$. A general and rigorous argument is not available, but we can utilize the approximate dielectric response function derived in the preceding paper $^{1}$ :

$$
\epsilon_{0} / \epsilon(k)=1-\kappa^{2} /\left\{k^{2}+\kappa^{2}[\cos (k a)-k \mu(k)]\right\},
$$

where

$\mu(k)=(4 \pi c)^{-1} \int d \mathbf{s} p^{(1)}(s) f(k, s)\left[I_{1}(k, s)-I_{2}(k, s)\right] ;$

$I_{1}(k, s)=\frac{\pi}{s} \int_{\max (a, s-a)}^{s+a} d t\left[a^{2}-(s-t)^{2}\right] \frac{\sin (k t)}{t}$

$I_{2}(k, s)=\frac{2 \pi}{s} \int_{0}^{a} d t[s+t-\max (a,|s-t|)] \sin (k t)$.

The "external field renormalization factor" $f(k, s)$ is a nonlinear functional of the ion-pair size distribution function $p^{(1)}$ :

$$
\begin{aligned}
f(k, s) & =\exp \left\{4 \pi k^{-1} \int_{0}^{s} t \sin (k t) p^{(1)}(t)[\rho(t)]^{-1} d t\right\}, \\
\rho(t) & =4 \pi \int_{t}^{\infty} u^{2} p^{(1)}(u) d u .
\end{aligned}
$$

If $\mu(k)$ were analytic in $k$ within some neighborhood of $k=0$, then it is clear from Eq. (3.5) that $\epsilon_{0} / \epsilon(k)$ would likewise be analytic at the origin. We shall proceed to make a small- $k$ evaluation of $\mu(k)$ to see if the result is consistent with analyticity.

It was argued in the preceding section that $p^{(1)}(s)$ has an inverse sixth-power "tail" for large $s$. For the time being it will be instructive to write the more general expression for $s \rightarrow \infty$ :

$$
p^{(1)}(s) \sim P s^{-v},
$$

where of course $P>0$ and $\nu>3$. We immediately have

Therefore,

$$
\rho(s) \sim 4 \pi P s^{3-\nu} /(\nu-3) .
$$

$$
\lim _{k \rightarrow 0} f(k, s)=c / \rho(s) \sim c(\nu-3) s^{\nu-3} / 4 \pi P .
$$

Due to occurrence of the "max" operation in both $I_{1}$ and $I_{2}$, these functions adopt different forms in the two intervals $a \leq s \leq 2 a$ and $2 a \leq s<\infty$. It is therefore convenient to split the $\mu(k)$ integral (3.6) into two parts corresponding to these $s$ ranges:

$$
\begin{aligned}
& \mu(k)=\mu_{1}(k)+\mu_{2}(k), \\
& \mu_{1}(k)=c^{-1} \int_{a}^{2 a} d s s^{2} p^{(1)} f\left[I_{1}-I_{2}\right], \\
& \mu_{2}(k)=c^{-1} \int_{2 a}^{\infty} d s s^{2} p^{(1)} f\left[I_{1}-I_{2}\right] .
\end{aligned}
$$

Because $\mu_{1}(k)$ involves integrals only between finite limits, it may be expanded in a convergent power series in $k$ simply by expanding the trigonometric functions in the integrands. Evidently then $\mu_{1}(k)$ is analytic at $k=0$.

On the other hand, $\mu_{2}(k)$ demands more delicate treatment for small $k$. When $2 a \leq s$ and $|s k| \ll 1$, we have

$$
\begin{aligned}
& I_{1}(k, s)-I_{2}(k, s) \\
& \quad=(\pi / s)\left\{\left[(4 / 45) a^{5}-(2 / 9) a^{3} s^{2}\right] k^{3}+O\left(k^{5}\right)\right\},
\end{aligned}
$$

whereas if $|s k| \gg 1$, the corresponding small- $k$ result is

$$
I_{1}(k, s)-I_{2}(k, s) \sim-\left(4 \pi a^{3} k / 3 s\right) .
$$

Evidently these two limiting forms effect a smooth connection in the range $|s k| \approx 1$. The external field renormalization factor $f(k, s)$ likewise exhibits different behavior for small and for large $|s k|$. Equation (3.8) shows in fact [consistent with (3.11)] that with fixed $s \geq 2 a$

$$
f(k, s) \sim c / \rho(s) \quad(|s k| \ll 1) ;
$$

on the other hand, if we write

$$
\begin{array}{r}
f(k, s)=\exp \left\{\frac{4 \pi}{k} \int_{a}^{s} t \sin (k t)\left[\frac{p^{(1)}(t)}{\rho(t)}-\frac{(\nu-3)}{4 \pi t^{3}}\right] d t\right. \\
\left.+\frac{\nu-3}{k} \int_{a}^{s} \frac{\sin (k t)}{t^{2}} d t\right\}
\end{array}
$$

(by adding and subtracting the relevant asymptote) the large $|s k|$ limit may be written

$$
\begin{aligned}
f(k, s) \sim \exp \left\{4 \pi \int_{a}^{\infty} t^{2}\right. & {\left[\frac{p^{(1)}(t)}{\rho(t)}-\frac{(\nu-3)}{4 \pi t^{3}}\right] d t } \\
& \left.+(\nu-3) \int_{|k| a}^{\infty} \frac{\sin u}{u^{2}} d u\right\}
\end{aligned}
$$


Since

$$
\int_{x}^{\infty} \frac{\sin u}{u^{2}} d u=-\ln x+1-\gamma+O\left(x^{2}\right)
$$

(where $\gamma=0.5772157 \cdots$ is the Euler-Mascheroni constant), we have

$$
\begin{aligned}
& f(k, s) \sim(|k| a)^{3-\nu} \exp \{(\nu-3)(1-\gamma) \\
&\left.+4 \pi \int_{a}^{\infty} t^{2}\left[\frac{p^{(1)}(t)}{\rho(t)}-\frac{(\nu-3)}{4 \pi t^{3}}\right] d t\right\} \\
& \equiv F(|k| a)^{3-\nu} \quad(|s k| \gg 1) .
\end{aligned}
$$

The different forms for $f$ suggested, respectively, by (3.15) and (3.19) must smoothly connect to one another when $|s k|$ is of order unity.

The integrand of expression (3.12) for $\mu_{2}(k)$ adopts distinctly different forms in the interior of the two ranges $2 a \leq s \leq|k|^{-1}$, and $|k|^{-1} \leq s<\infty$. In the former, Eqs. (3.13) and (3.15) imply

$$
\begin{aligned}
s^{2} p^{(1)} f\left[I_{1}-I_{2}\right] & \sim s^{2} P s^{-\nu}[c(\nu-3) / 4 \pi P] s^{\nu-3}\left[-\frac{2}{9} \pi a^{3} s k^{3}\right] \\
& =-[c(\nu-3) / 18] k^{3} a^{3},
\end{aligned}
$$

where we have invoked large-s asymptotes as having predominant importance in the large interval dictated by small $|k|$. In the latter range the analogous expression is:

$$
\begin{gathered}
s^{2} p^{(1)} f\left[I_{1}-I_{2}\right] \sim s^{2} P s^{-\nu} F(|k| a)^{3-\nu}\left[-4 \pi a^{3} k / 3 s\right] \\
=-\frac{4}{3} \pi P F a^{6-\nu} s^{1-\nu} k|k|^{3-\nu} .
\end{gathered}
$$

The $\mu_{2}(k)$ integral may now be estimated, up to a positive factor of order unity, by integrating (3.20) and (3.21) over the respective intervals ${ }^{9}$ :

$$
\begin{aligned}
\mu_{2}(k) & \approx-\frac{(\nu-3)}{18} \int_{0}^{|k|^{-1}} k^{3} a^{3} d s-\frac{4 \pi F}{3}\left(\frac{P a^{3-\nu}}{c}\right) \\
& \times \int_{|k|^{-1}}^{\infty} a^{3} k|k|^{3-\nu} s^{1-\nu} d s \\
& =-[(\nu-3) / 18] a^{3} k|k|-[4 \pi F / 3(\nu-2)] a^{3} k|k| \\
& \equiv-M a^{3} k|k|,
\end{aligned}
$$

where $M$ is a positive dimensionless number of order unity. Since we can easily show that $\mu_{1}(k)$ is $O\left(k^{3}\right)$ for small $k$, we have

$$
\mu(k)=-M a^{3} k|k|+O\left(k^{3}\right) .
$$

The occurrence of $|k|$ in this result shows that $\mu(k)$ cannot be analytic in the neighborhood of $k=0$. Although $\mu(k)$ is manifestly an odd function of $k$ :

$$
\mu(-k)=-\mu(k),
$$

an analytic continuation of this function from the positive real axis onto the negative real axis (if such a continuation is possible) will not agree with (3.24)
The simplest possible $\mu(k)$ would be composed of two analytic functions, the individual continuations off the positive and negative portions of the real $k$ axis, which are separated by a branch cut along the entire imaginary $k$ axis.

Return now to Eq. (1.2), which gives essentially the distribution of charge in the ion atmospheres, and in which we now insert the explicit $\epsilon_{0} / \epsilon(k)$ expression (3.5):

$$
\begin{aligned}
g_{+-}{ }^{(2)}(r)-g_{+} & +(2)(r)=\left(4 \pi^{2} c r\right)^{-1} \int_{-\infty}^{+\infty} d k k \sin (k r) \\
& \times\left\{1-\frac{k^{2}}{k^{2}+k^{2}[\cos (k a)-k \mu(k)]}\right\}
\end{aligned}
$$

If $\mu(k)$ were meromorphic, this integral would be amenable to evaluation by Cauchy's integral theorem, and the ion atmosphere would be composed of a series of contributions exponentially damped in $r$. However now we must write

$$
\begin{aligned}
g_{+-}{ }^{(2)}(r)- & g_{++}{ }^{(2)}(r)=\left(4 \pi^{2} c r\right)^{-1} \int_{-\infty}^{+\infty} d k k \sin (k r) \\
& \times\left\{1-\frac{k^{2}}{k^{2}+k^{2} \cos (k a)}+k^{-1} \Re(k)\right\},
\end{aligned}
$$

where $\mathfrak{M}(k)$ is nonanalytic at the origin

$$
\begin{aligned}
\mathscr{T}(k)=k^{3}\left(\left[k^{2}+k^{2} \cos (k a)\right]^{-1}\right. \\
\left.-\left\{k^{2}+k^{2}[\cos (k a)-k \mu(k)]\right\}^{-1}\right) .
\end{aligned}
$$

One can conclude from Eq. (3.23) that

$$
\mathfrak{T}(k)=\left(M a^{3} / \kappa^{2}\right) k^{5}|k|+O\left(k^{6}\right) .
$$

The Cauchy integral theorem applies to the terms preceding $k^{-1} \mathfrak{T}(k)$ in Eq. (3.26), and they will provide exponentially damped contributions to the ion atmosphere. For the $\mathfrak{T}(k)$ term, however, we must appeal to the existing theory of asymptotic Fourier integral evaluation ${ }^{10}$ to conclude that for large $r$ :

$$
\int_{-\infty}^{+\infty} d k \sin (k r) \operatorname{Tr}(k) \sim-\frac{1440 M a^{3}}{\kappa^{2} r^{7}} .
$$

The corresponding behavior of the ion atmosphere is

$$
g_{+}-^{(2)}(r)-g_{++}{ }^{(2)}(r) \sim-\left(360 M a^{3} / c \pi^{2} \kappa^{2} r^{8}\right) .
$$

The nonanalytic character of $\mu(k)$ has thus produced an algebraic tail in the ion atmosphere charge distribution.

The details in Eq. (3.22) indicate that the positive constant $M$ could vary with $\nu$, although not in such a way that it could vanish. It is interesting to note, however, that this is the only way that $\nu$ affects result (3.30), so in fact the $r^{-8}$ tail in the ion atmosphere does not hinge sensitively on the assumption that $\nu=6$.

The leading small- $k$ term shown in Eq. (3.28) for $\mathfrak{T}(k)$ is alone a valid representation at low concentra- 
tion only if $|k| \ll \kappa$. This means in turn that the $r^{-8}$ tail in (3.30) applies only for large $\kappa r$. Hence writing

$$
g_{+}-^{(2)}(r)-g_{++}{ }^{(2)}(r) \sim-\left(360 M \kappa^{6} a^{3} / \pi^{2} c\right)(\kappa r)^{-8} \text {, }
$$

we can see that the "strength" of the tail expressed in dimensionless distance units declines as $c^{2}$ at low concentration. $\operatorname{Ir}(k)$ is not involved at all in the low concentration limit; the linear Debye-Hückel theory arises entirely from the other terms in Eq. (3.26) which are meromorphic.

The ion-pair theory of electrolytes in its present form does not separately specify $g_{+-{ }^{(2)}}(\boldsymbol{r})$ and $g_{++}{ }^{(2)}(\boldsymbol{r})$. However if our $r^{-8}$ conclusion is correct for the difference of these functions, it seems reasonable to suppose that

$$
\begin{aligned}
& g_{+-}{ }^{(2)}(r)-1 \sim-\left(360 M_{1} a^{3} / \pi^{2} c \kappa^{2} r^{8}\right), \\
& g_{++}{ }^{(2)}(r)-1 \sim\left(360 M_{2} a^{3} / \pi^{2} c \kappa^{2} r^{8}\right),
\end{aligned}
$$

where both $M_{1}$ and $M_{2}$ are positive $\left(M=M_{1}+M_{2}\right)$.

\section{DISCUSSION}

The function $\mu(k)$ which has been implicated in the $r^{-8}$ tail arises in the $\epsilon_{0} / \epsilon(k)$ calculation of the preceding paper $^{1}$ as an electrostatic interaction between an ion and induced charge in a spherical region of ionic size. The infinite upper $s$ limit on $\mu_{2}$ in Eq. (3.12) is thus associated with the infinite range of the Coulomb interaction. If instead the Coulomb law involved an upper cutoff distance $R$ (beyond which ions did not interact), the $\mu_{2}$ integral would have a corresponding upper limit. The asymptotic small- $k$ analysis of $\mu_{2}$ then would be fundamentally altered, and when $|k|^{-1}>R$, Eq. (3.22) would have to read

$$
\begin{aligned}
\mu_{2}(k) & \approx \int_{0}^{R}\left[-\frac{1}{18}(\nu-3) k^{3} a^{3}\right] d s \\
& =-\frac{1}{18}(\nu-3) R a^{3} k^{3} .
\end{aligned}
$$

Nonanalyticity is not indicated. The evidence thus implies that the algebraic tail obtained for the ion atmosphere charge density is intimately connected with the long-range character of the Coulomb interaction.

One of the more popular techniques for study of the statistical mechanics of electrolytes is the Ursell-Mayer cluster expansion. ${ }^{11}$ The long range of the Coulomb potential causes large classes of the cluster integrals for electrolyte thermodynamic properties to diverge. Consequently, considerable cleverness had to be exercised to identify that class of divergent clusters ("chain diagrams") whose formal infinite-order summation would reproduce the Debye-Hückel limit. ${ }^{12}$ Historically, of course, the Debye-Hückel theory was developed far earlier, on a noncluster-theoretic basis.

Chain diagram summations have also formed the basis for electrolyte cluster series rearrangements that have been directed to understanding concentrated electrolytes. Unfortunately, the cluster integral orderof-magnitude estimates that have been advanced in support of these rearrangements are far from rigorous mathematically. ${ }^{11-13}$ In any event their genesis seems strongly to have been influenced by the topological flavor of the Debye-Hückel limit.

Analogous chain diagram summations have also been advocated for concentrated electrolyte pair correlation functions. ${ }^{14}$ For computational purposes it has proven convenient to utilize generating functionals to effect the chain summations implicitly. These functionals appear in the form of nonlinear integral equations, and are now conventionally referred to as the "hypernetted chain" (HNC) integral equations..$^{15,16}$

The chain summations incorporated into the HNC integral equations tend to replace the original Mayer $f$ bonds (which are long ranged due to the Coulomb interaction) with an exponentially shielded effective bond. The predicted ionic correlation functions in turn possess such exponential damping. Still, the mathematical danger involved in arbitrary rearrangement of divergent series (indeed in this case series with divergent terms!) is well known, so the usual chain summations may be quite unjustified beyond the verification of the Debye-Hückel limit. It is not inconceivable that some rearrangement of the complete formal $g_{i j}{ }^{(2)}$ cluster series which specifically involves clusters with high connectivity ("bridging") could produce $r^{-8}$ asymptotic decay.

Integral equations of the Kirkwood ${ }^{17}$ and of the Born-Green-Yvon ${ }^{18}$ types have also been frequently used to investigate ionic pair correlation in electrolytes. Such integral equations in their exact versions involve higher-order distribution functions which are unknown, so one is always forced to invoke a superposition approximation $^{19}$ to express these higher-order quantities as simple functionals of the pair functions. It is apparently the case that these approximate integral equations always predict exponentially damped pair correlations. However it is also the case that the superposition error might have precisely such a character that if it were formally reintroduced into the integral equations, their solutions would be forced to possess $r^{-8}$ tails. For electrolytes, then, it may well be that superposition approximations are especially inappropriate out of the Debye-Hückel dilute limit.

Analogous comments apply to use of the PercusYevick integral equations in the electrolyte problem. ${ }^{15}$ With this approach one is obliged to approximate the so-called "direct correlation functions" in terms of the ionic potentials and pair correlation functions. The Percus-Yevick prescription produces direct correlation functions which are as long ranged as the Mayer $f$ functions, so that as in the HNC case one effectively sums chains to exponentially damped pair correlations. But the exact direct correlation functions could well have a different asymptotic large-distance behavior from the Mayer $f$ 's (possibly involving some inverse fractional 
power), and the way is again open for $r^{-8}$ tails in the various $g_{i j}{ }^{(2)}$.

In conclusion, there seems to be no present logical basis for maintaining that electrolyte pair correlation remains exponentially damped at finite concentrations. Our ion-pair theory suggests that a subtle interplay exists between short-ranged core repulsions between ions, and their long-ranged Coulombic interactions which makes the static dielectric response nonanalytic. The implied algebraic correlation tail is one worth looking for by alternative theoretical methods, but only if special care is devoted to avoiding approximation procedures which are from the outset functionally inconsistent with the existence of such a tail.

* Present address: Departments of Mathematics and Computing Sciences, University of Southwestern Louisiana, Lafayette, La. 70501 .

${ }^{1}$ F. H. Stillinger and R. J. White, J. Chem. Phys. 54, 3395 (1971), Preceding paper, Paper III.

${ }^{2}$ P. Debye and E. Hückel, Physik. Z. 24, 185, 305 (1923).

3 J. C. Poirier, in Chemical Physics of Ionic Solutions, edited by B. E. Conway and R. G. Barradas, (Wiley, New York, 1966), p. 9. See also D. N. Card and J. P. Valleau, J. Chem. Phys. 52, $6232(1970)$.

${ }^{4}$ The exceptional ambiguous cases with equal pair distances have zero probability, and may be disregarded.

${ }^{5} \mathrm{~F}$. H. Stillinger and R. Lovett, J. Chem. Phys. 48, 3858 (1968).

${ }_{6}^{6}$ R. Lovett and F. H. Stillinger, J. Chem. Phys. 48, 3869 (1968).

${ }_{7}^{7}$ J. G. Kirkwood, J. Chem. Phys. 7, 911 (1939).

8 The constant $C\left(s_{0}\right)$ is required to maintain normalization of $p^{(1)}(s)$, and $s_{0}$ is arbitrary.

${ }^{9}$ Use of the lower integration limit 0 (rather than $2 a$ ) causes no error in our leading-order estimate.

${ }_{10} \mathrm{M}$. J. Lighthill, An Introduction to Fourier Analysis and Generalized Functions (Cambridge U. P., Cambridge, England, 1962), Chap. 4.

${ }^{11}$ H. L. Friedman, Ionic Solution Theory (Interscience, New York, 1962).

12 J. E. Mayer, J. Chem. Phys. 18, 1426 (1950).

${ }_{13}$ P. M. V. Résibois, Electrolyte Theory (Harper and Row, New York, 1968), pp. 123-128.

${ }^{14}$ E. Meeron, J. Chem. Phys. 28, 630 (1958).

${ }^{15}$ D. D. Carley, J. Chem. Phys. 46, 3783 (1967).

${ }_{16}$ J. C. Rasaiah and H. L. Friedman, J. Chem. Phys. 48, 2742

(1968); J. C. Rasaiah and H. L. Friedman, ibid. 50, 3965 (1969).

${ }_{17}$ J. G. Kirkwood and J. C. Poirier, J. Phys. Chem. 58, 591 (1954).

${ }^{18}$ C. W. Outhwaite, J. Chem. Phys. 50, 2277 (1969).

${ }_{19}$ T. L. Hill, Statistical Mechanics (McGraw-Hill, New York, 1956), Chap. 6.

\title{
Modes and Rates of Reaction of Cyclopentene and Methylcyclopentene Ions with Their Parent Molecules*
}

\author{
R. Lesclaux $\nmid \dagger$ S. Searles, $\ddagger$ L. Wayne Sieck, and P. Ausloos \\ National Bureau of Standards, Washington, D. C. 20234
}

(Received 2 October 1970)

\begin{abstract}
Cyclopentene and methylcyclopentene ions were generated by irradiating the respective parent compounds with $10.0-\mathrm{eV}$ photons; cyclopentene was also irradiated with $11.6-11.8-\mathrm{eV}$ photons. The ionic products of ion-molecule reactions were observed in the NBS high-pressure photoionization mass spectrometer, while the neutral products of these reactions were determined by chemical analysis of products formed in photolytic experiments in a closed system. The cyclopentene ion, which at $10.0 \mathrm{eV}$ retains its cyclic structure, undergoes an $\mathrm{H}_{2}$ transfer reaction $\left(c-\mathrm{C}_{5} \mathrm{H}_{8}{ }^{+}+c-\mathrm{C}_{6} \mathrm{H}_{8} \rightarrow c-\mathrm{C}_{5} \mathrm{H}_{10}+c-\mathrm{C}_{5} \mathrm{H}_{6}{ }^{+}, k=3.3 \times 10^{-10} \mathrm{~cm}^{3}\right.$ ) molecule $\cdot \mathrm{sec})$ and a condensation reaction $\left(c-\mathrm{C}_{5} \mathrm{H}_{8}^{+}+c-\mathrm{C}_{5} \mathrm{H}_{8} \rightarrow \mathrm{C}_{10} \mathrm{H}_{16}, k=2.7 \times 10^{-10} \mathrm{~cm}^{3} / \mathrm{molecule} \cdot \mathrm{sec}\right)$ with the parent molecule. The same reactions are observed for ions formed at $11.6-11.8 \mathrm{eV}$, but at the higher energy, approximately $20 \%$ of the ions are observed to undergo ring opening to form $1,3-\mathrm{C}_{5} \mathrm{H}_{8}{ }^{+}$ions; the latter ions undergo an $\mathrm{H}_{2}$ transfer reaction with the cyclopentene molecule $\left(1,3-\mathrm{C}_{5} \mathrm{H}_{8}{ }^{+}+c-\mathrm{C}_{5} \mathrm{H}_{8} \rightarrow\right.$ $2-\mathrm{C}_{5} \mathrm{H}_{10}+\mathrm{C}_{5} \mathrm{H}_{6}^{+}, k=1 \times 10^{-10} \mathrm{~cm}^{3} /$ molecule $\left.\cdot \mathrm{sec}\right)$. The three methylcyclopentene ions also undergo $\mathrm{H}_{2}$ transfer reactions with the corresponding parent molecules $\left[\mathrm{C}_{6} \mathrm{H}_{10}{ }^{+}+\mathrm{C}_{6} \mathrm{H}_{10} \rightarrow\left(\mathrm{CH}_{3}\right) \mathrm{C}_{5} \mathrm{H}_{9}+\mathrm{C}_{6} \mathrm{H}_{8}{ }^{+}, k=4.6\right.$, 1.8 , and $2.9 \times 10^{-10} \mathrm{~cm}^{3} /$ molecule $\cdot \mathrm{sec}$ for 1-, 3-, and 4-methylcyclopentene, respectively]. Condensation reactions between these ions and their parent molecules are observed to only a minor extent, but a second major mode of reaction, namely $\mathrm{H}$ transfer, is observed $\left(\mathrm{C}_{6} \mathrm{H}_{10}{ }^{+}+\mathrm{C}_{6} \mathrm{H}_{10} \rightarrow \mathrm{C}_{6} \mathrm{H}_{11}+\mathrm{C}_{6} \mathrm{H}_{9}{ }^{+}, k=0.6,2.8\right.$, and $1.2 \times 10^{-10} \mathrm{~cm}^{3} / \mathrm{molecule} \cdot \mathrm{sec}$ for 1 -, 3-, and 4-methylcyclopentene, respectively). Structural reasons for these rate variations are discussed.
\end{abstract}

\section{INTRODUCTION}

In a recent study from this laboratory, ${ }^{1}$ the reactions of the cyclohexene parent ion were examined in a photoionization mass spectrometer and also by analysis of the neutral products formed through the chemical reactions of ions formed in the closed-system photolysis of cyclohexene at energies above the ionization energy. In the mass spectrometer, where the ions were formed by irradiating cyclohexene with 10.0 - and $11.6-11.8-\mathrm{eV}$ photons, it was seen that the cyclohexene parent ion reacts with cyclohexene to form $\mathrm{C}_{6} \mathrm{H}_{8}{ }^{+}$and $\mathrm{C}_{12} \mathrm{H}_{20}{ }^{+}$as product ions,

$$
\begin{gathered}
\mathrm{C}_{6} \mathrm{H}_{10}{ }^{+}+\mathrm{C}_{6} \mathrm{H}_{10} \rightarrow \mathrm{C}_{6} \mathrm{H}_{12}+\mathrm{C}_{6} \mathrm{H}_{8}{ }^{+}, \\
\mathrm{C}_{6} \mathrm{H}_{10}{ }^{+}+\mathrm{C}_{6} \mathrm{H}_{10} \rightarrow \mathrm{C}_{12} \mathrm{H}_{20}{ }^{+} .
\end{gathered}
$$

It could be derived from the results that at the higher 